\title{
Using Ultrasound Guided Needle Biopsy in Combination With GeneXpert MTB/RIF to Diagnose Epididymal Tuberculosis: a Study of 12 Cases
}

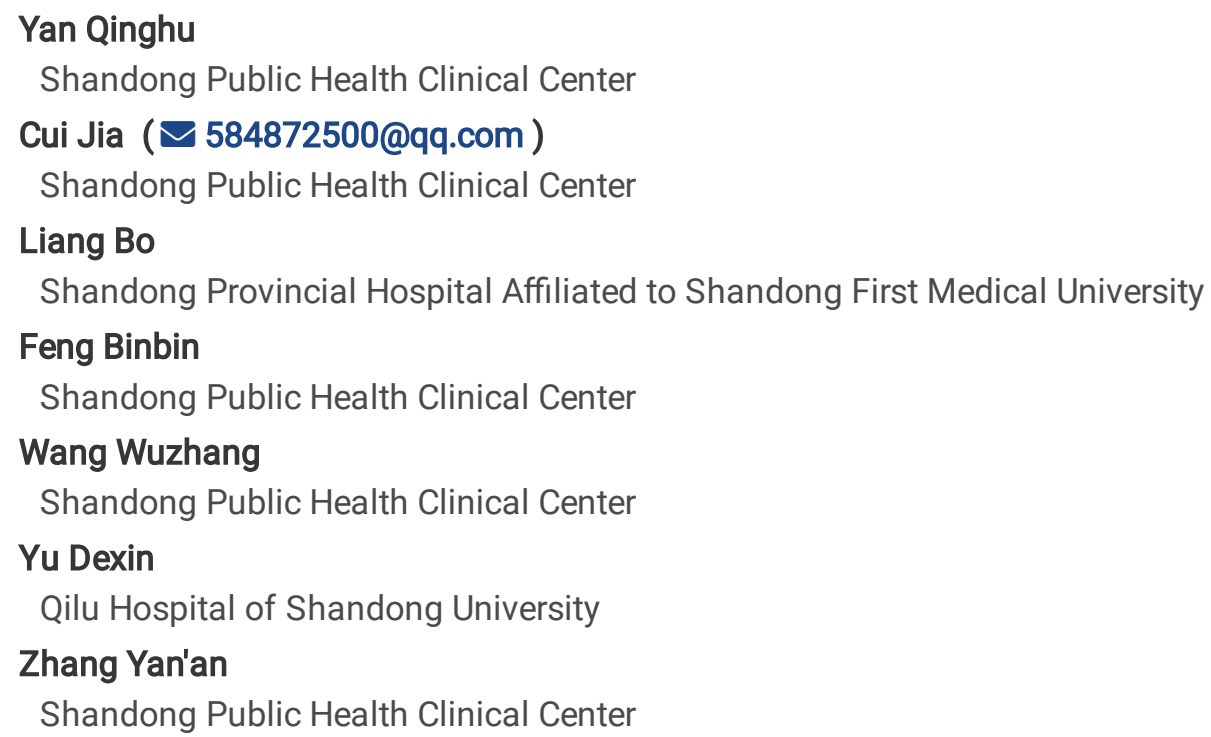

Yan Qinghu

Shandong Public Health Clinical Center

Cui Jia (D584872500@qq.com )

Shandong Public Health Clinical Center

Liang Bo

Shandong Provincial Hospital Affiliated to Shandong First Medical University

Feng Binbin

Shandong Public Health Clinical Center

\section{Wang Wuzhang}

Shandong Public Health Clinical Center

\section{Yu Dexin}

Qilu Hospital of Shandong University

\section{Zhang Yan'an}

Shandong Public Health Clinical Center

\section{Research Article}

Keywords: Epididymal tuberculosis, Needle biopsy, Ultrasonic guidance, GeneXpert MTB/RIF

Posted Date: January 6th, 2022

DOI: https://doi.org/10.21203/rs.3.rs-952085/v1

License: (c) (i) This work is licensed under a Creative Commons Attribution 4.0 International License. Read Full License 


\section{Abstract}

Objective: This study aims to investigate the diagnostic utility of percutaneous ultrasound-guided needle biopsy in combination with GeneXpert MTB/RIF for epididymal tuberculosis.

Methods: Between March 2019 to March 2021, specimens obtained from 12 patients with confirmed epididymal tuberculosis by ultrasound guided needle biopsy were sent for pathology and laboratory examination at the Shandong Public Health Clinical Center. The laboratory examination included acid-fast staining, Mycobacterium tuberculosis culture by BACTEC MGIT 960, and the GeneXpert MTB/RIF test. The diagnosis and complications were comprehensively analyzed.

Results: Among the 12 cases, seven cases had granulomatous inflammation and necrotic tissue, four cases had chronic inflammatory cells and necrotic tissue, and one case had chronic inflammatory cell infiltration. Furthermore, among the 12 patients, five patients were positive for Mycobacterium tuberculosis culture, two patients were positive for the acid-fast staining, and twelve patients were positive for the GeneXpert MTB/RIF assay. The sensitivity and specificity of the acid-fast staining, Mycobacterium tuberculosis culture and GeneXpert MTB/RIF in the diagnosis of epididymis tuberculosis were $16.67 \%$ and $100.00 \%, 41.67 \%$ and $100.00 \%$, and $100.00 \%$ and $50.00 \%$, respectively. The diagnostic value analysis of the three detection techniques indicated that the GeneXpert MTB/RIF technique (Kappa=0.63, AUC $=0.75$ ) is superior to the Mycobacterium tuberculosis culture (Kappa=0.17, AUC $=0.71)$ and acid-fast staining $(\mathrm{Kappa}=0.05, \mathrm{AUC}=0.58)$.

Conclusion: Ultrasound guided percutaneous biopsy combined with GeneXpert MTB/RIF assay is extremely useful for diagnosing epididymitis tuberculosis and determining rifampin resistance, with superior sensitivity, specificity and AUC value.

\section{Introduction}

Extrapulmonary tuberculosis accounts for $10 \%$ of all tuberculosis cases [1]. Urogenital tuberculosis accounts for $30 \%-40 \%$ of extrapulmonary tuberculosis cases, with epididymal tuberculosis being even more uncommon [2,3]. Due to its insidious onset and mild symptoms, early epididymal tuberculosis can easily be missed [4]. The epididymis can be divided into three sections: the head, body and tail. Furthermore, it primarily comprises of epididymal tubules that migrate to the tail's vas deferens. Mycobacterium tuberculosis can enter the prostate and seminal vesicles via urine, and retrograde through the vas deferens, forming irregular masses at the epididymal tail. Then, the epididymal body and head are subsequently invaded, causing the entire epididymis to swell, and the testis is finally invaded. This can also be caused by the primary infection transmitted through the bloodstream to the epididymis. In clinic, epididymal tuberculosis is very clinically similar to testicular tumors, testicular torsion and testicular bacterial inflammation, making it difficult to determine a definitive diagnosis under present medical conditions [5-6]. Ultrasound has the advantages of fast, simple and no radiation, and it can be used for both the primary screening and advance detection of epididymal tuberculosis [7]. In the early stage of tuberculosis, the caseous lesions in the nodules are small, which mainly manifest as exudation and hyperplasia, and the blood flow signals are more abundant. If the infection develops further, necrosis forms in the lesion center and peripheral microvessels dilate, presenting abundant peripheral blood flow signals. As the lesion develops, the caseous necrosis area becomes enlarged and fused, most of the normal tissue structure becomes destroyed, and the lesion area presents with a decreased signal or even no signal.

However, since the ultrasonic manifestations of epididymal tuberculosis are very similar to the manifestations of epididymal tumors, epididymal granuloma and non-specific epididymitis, pathological and microbial examinations are still required to confirm the definitive diagnosis [8]. Due to its real-time, accurate and safe characteristics, ultrasound guided puncture can be used for both pathological biopsy and laboratory examinations, in order to confirm the diagnosis. Real-time fluorescence quantitative nucleic acid amplification for rifampin resistance (GeneXpert MTB/RIF) is a relatively novel tuberculosis diagnosis technology [9], which has higher sensitivity and specificity, when compared to traditional tuberculosis tests, and this can be used for rifampin resistance detection. The present study aims to determine the utility of ultrasound-guided percutaneous epididymal puncture in conjunction with GeneXpert MTB/RIF for diagnosing epididymal tuberculosis.

\section{Materials And Methods}




\section{Study subjects}

A total of 14 patients with clinically suspected epididymal tuberculosis, who were admitted to our hospital from June 2019 to March 2021, were enrolled in the present study. The content of this study was approved by the ethics committee of Shandong Public Health Clinical Center. All methods were carried out in accordance with relevant guidelines and regulations. All patients underwent pathological and laboratory examinations with the ultrasound-guided puncture biopsy of specimens. Then, the clinical symptoms and ultrasound characteristics were combined for comparison and analysis. According to the World Health Organization (WHO) guidelines [10] and Chinese Medical Association Tuberculosis Clinical Diagnostic Criteria [11], clinically diagnosed tuberculosis patients must meet the following criteria: (1) clinical symptoms consistent with tuberculosis; (2) clinical imaging highly suggestive of tuberculosis; (3) effective anti-tuberculosis treatment. All lesions were routinely examined by ultrasound prior to the needle biopsy. The information of the patients were retrieved from clinical records, which included age, gender, comorbidities, laboratory tests, and response to treatment. None of these patients received anti-tuberculosis therapy prior to the biopsy. This study was approved by the ethics committee of Shandong Public Health Clinical Center (Shandong Chest Hospital) (2021XKYYEC-22). The study is in line with the declaration of Helsinki.

\section{Ultrasound guided puncture}

The Philips Q7c ultrasonic diagnostic instrument was used for the ultrasound examination, with a probe frequency of 5-12 $\mathrm{MHz}$. All biopsies were collected under ultrasound guidance by an ultrasound surgeon experienced in performing punctures. Briefly, the patient was placed in the supine position, according to the location of the lesion. The biopsy site was routinely disinfected, sterile toweled, and applied with local layered anesthesia with $2 \%$ lidocaine hydrochloride. A semi-automatic cutting biopsy needle ( $18 \mathrm{G}, 10 \mathrm{~cm}$; BD, USA) was used to slowly puncture the lesion for 2-3 times under continuous real-time ultrasound monitoring. (Figure 1). For all patients, these biopsy specimens were subjected to pathological examination, acidfast staining, BACTEC MGIT 960 Mycobacterium tuberculosis culture, and GeneXpert MTB assay.

\section{Pathological examination}

The epididymis tissues were fixed with $10.0 \%$ formalin and embedded in paraffin before being sequentially sectioned. Sections with a thickness of $4 \mu \mathrm{m}$ were stained with $\mathrm{H} \& \mathrm{E}$, and observed by light microscopy by two experienced pathologists. Under light microscopy, the typical lesions of tuberculosis, such as epithelioid granulomas, Langhans giant cells and caseous necrosis, as well as other lesions, such as fibrosis and calcification fibrosis, were visible in the pathological tissue. This is consistent with the pathological characteristics of tuberculosis.

\section{Pathogen tests}

The acid-fast staining method (Zhuhai Basso, China) and modified alkaline compound red method were used, strictly according to manufacturer's instructions. The BACTEC MGIT 960 (BD, USA) mycobacterium culture monitoring system and supporting reagents were used, and the strains were identified according to the instructions and specifications. After adding an appropriate amount of phosphate buffer, the pathological tissues were homogenized using the micro-grinder FAST-PREP-24 (MP Biomeidcals, USA), and these were subsequently used for GeneXpert MTB/RIF detection (Cepheid, USA) [12], which is based on semi-nested PCR and automated fluorescence detection [13].

\section{Statistical analysis}

All data were processed in Excel 2010 and statistically analyzed using SPSS 24.0. Normally distributed data were expressed as mean and standard deviation $(X \pm S D)$, while median and quartile spacing $(M \pm I Q R)$ were used to express non-normally distributed data. The receiver operating characteristic (ROC) curve, area under the ROC curve (AUC), KAPPA value, sensitivity, specificity, positive predictive value and negative predictive value were used for statistical inference. $P<0.05$ was considered statistically significant.

\section{Results}


The age of the patients ranged within 31-76 years old, with a mean age of $46.3 \pm 18.0$ years old. The longest duration of disease was two years, and the shortest duration was three months. Among the 12 cases of epididymal tuberculosis confirmed in the present study, seven patients (58.3\%) had coexisting tuberculosis of another organ. Furthermore, for these 12 cases, the clinical manifestations of 11 cases (91.7\%) were scrotal mass or scrotal pain, and falling sensation, while the clinical manifestations of the remaining case (8.3\%) were fever and night sweats. There were no complications in any of the 14 patients. However, one patient $(8.3 \%, 1 / 12)$ had mild local bleeding, which was resolved after the operation was stopped.

\section{Ultrasonic manifestations}

All 12 patients had varying degrees of epididymal changes: nine patients had lesions in the tail, two patients had lesions in both the head and tail, and one patient had lesions in the head, body and tail. The two-dimensional ultrasound revealed that the lesions in the epididymis had a heterogeneous echo area. In nine patients, the volume of the epididymis increased, the lesions were mainly in the tail, the boundary was unclear, the internal echo decreased, and the color Doppler results revealed a decrease in blood flow signal. In the seven cases with testicular involvement, the ultrasonography revealed a testicular mass with a low blood flow signal. Five of these cases had scrotal swelling, thickening of the scrotal wall, irregular hypoecho in the scrotum, visible sinus tract, and significantly reduced or even no blood flow signal in the color Doppler lesions. Furthermore, three cases presented with scrotal ulceration. Yang et al. [14] considered that the decreased blood flow signal in the lesion was caused by caseous necrosis and calcification, which led to the destruction of the vascular structure.

\section{Pathological abnormalities}

In the present study, the ultrasonography of the epididymal tuberculosis revealed a variety of pathological changes. Among the 12 patients, seven patients had granulomatous inflammation and necrotic tissue, four patients had chronic inflammatory cells with necrotic tissue, and one patient had chronic inflammatory cell infiltration. Since these were detected in these patients relatively early, there was no calcification.

\section{Laboratory results}

For the 12 patients, five patients $(41.67 \%, 5 / 12)$ were positive for the MGIT960 Mycobacterium tuberculosis culture, two patients $(16.67 \%, 2 / 12)$ were positive for the acid-fast staining, and 12 patients $(100 \%, 12 / 12)$ were positive for the GeneXpert MTB/RIF assay, which had the highest positivity rate (Table 1). The specificity of the acid-fast staining, tuberculosis culture and GeneXpert MTB/RIF technique in the diagnosis of epididymis tuberculosis was $100.00 \%, 100.00 \%$ and $50.00 \%$, respectively. In addition, all cases with a positive Mycobacterium tuberculosis culture were also positive for the GeneXpert MTB/RIF assay. However, the BACTEC MGIT 960 and histopathological results were negative for two confirmed patients. As shown in Table 2, GeneXpert MTB/RIF improved the pathogen detection rate of tissue biopsy specimens by $16.67 \%$ (2/12). The diagnostic value for epididymis tuberculosis was, as follows: GeneXpert MTB/RIF technique (AUC=0.75) > Mycobacterium tuberculosis culture $(A U C=0.71)$ > acid-fast staining (AUC=0.58) (Table 2, Figure 2). However, the KAPPA consistency test revealed that GeneXpert MTB/RIF had general consistency (KAPPA $=0.63)$, while the acid-fast staining (KAPPA $=0.05)$ and tuberculosis culture (KAPPA=0.17) had poor consistency(Table 2). 
Table 1

Diagnostic results of pathology and the three laboratory methods for epididymal tuberculosis $(n)$

\begin{tabular}{|c|c|c|c|c|}
\hline \multirow[t]{2}{*}{ Detection technique } & \multirow[t]{2}{*}{ Detection result } & \multicolumn{3}{|c|}{ Clinical comprehensive diagnosis } \\
\hline & & Epididymal tuberculosis & Non-epididymal tuberculosis & Total \\
\hline \multirow[t]{3}{*}{ Pathology } & Positive & 7 & 0 & 7 \\
\hline & Negative & 5 & 2 & 7 \\
\hline & Total & 12 & 2 & 14 \\
\hline \multirow[t]{3}{*}{ Acid-fast staining } & Positive & 2 & 0 & 2 \\
\hline & Negative & 10 & 2 & 12 \\
\hline & Total & 12 & 2 & 14 \\
\hline \multirow{3}{*}{$\begin{array}{l}\text { Mycobacterium } \\
\text { tuberculosis culture }\end{array}$} & Positive & 5 & 0 & 5 \\
\hline & Negative & 7 & 2 & 9 \\
\hline & Total & 12 & 2 & 14 \\
\hline \multirow[t]{3}{*}{ GeneXpert MTB/RIF } & Positive & 12 & 1 & 13 \\
\hline & Negative & 0 & 1 & 1 \\
\hline & Total & 12 & 2 & 14 \\
\hline
\end{tabular}

Table 2

Diagnostic value of pathology and the three laboratory methods for epididymal tuberculosis

\begin{tabular}{|llllllllll|}
\hline $\begin{array}{l}\text { Detection } \\
\text { techniques }\end{array}$ & AUC & $\begin{array}{l}\text { Sensitivity } \\
\text { (\%) }\end{array}$ & $\begin{array}{l}\text { Specificity } \\
(\%)\end{array}$ & $\begin{array}{l}\text { Positive } \\
\text { predictive } \\
\text { value (\%) }\end{array}$ & $\begin{array}{l}\text { Negative } \\
\text { predictive } \\
\text { value (\%) }\end{array}$ & $\begin{array}{l}\text { Kappa } \\
\text { value }\end{array}$ & $\begin{array}{l}\text { Total } \\
\text { coincidence } \\
\text { rate (\%) }\end{array}$ & $\begin{array}{l}\text { Jordan } \\
\text { index }\end{array}$ \\
\hline Pathology & 0.792 & $58.33 \%$ & $100.00 \%$ & $100.00 \%$ & $28.57 \%$ & 0.286 & 0.127 & $64.29 \%$ & $58.33 \%$ \\
\hline $\begin{array}{l}\text { Acid-fast } \\
\text { staining }\end{array}$ & 0.583 & $16.67 \%$ & $100.00 \%$ & $100.00 \%$ & $16.67 \%$ & 0.054 & 0.533 & $28.57 \%$ & $16.67 \%$ \\
\hline $\begin{array}{l}\text { Mycobacterium } \\
\text { tuberculosis } \\
\text { culture }\end{array}$ & 0.708 & $41.67 \%$ & $100.00 \%$ & $100.00 \%$ & $22.22 \%$ & 0.169 & 0.255 & $50.00 \%$ & $41.67 \%$ \\
\hline $\begin{array}{l}\text { GeneXpert } \\
\text { MTB/RIF }\end{array}$ & 0.750 & $100.00 \%$ & $50.00 \%$ & $92.31 \%$ & $100.00 \%$ & 0.632 & 0.011 & $92.86 \%$ & $50.00 \%$ \\
\hline
\end{tabular}

\section{Discussion}

Pulmonary tuberculosis is mainly tuberculosis, and is mainly confirmed through respiratory tract specimens. For patients without any respiratory symptoms, the diagnosis of extrapulmonary tuberculosis is extremely difficult when using common laboratory and imaging tests. The tuberculosis of the male reproductive system is a kind of extrapulmonary tuberculosis that most commonly affects the epididymis, seminal vesicles, prostate and testis. Due to its rich blood supply, the caudal epididymis is the first to be affected with the most serious lesion. Since the vas deferens runs alongside the tail of the epididymis, the Mycobacterium tuberculosis can easily spread directly along the vas deferens to the tail of the epididymis [15, 16]. Microscopically, multiple tuberculous granulomas of varying sizes can be observed in swelling epididymal tissues with extensive caseous necrosis, and these are frequently accompanied by significant acute or chronic nonspecific inflammation. Kim et al. considered that epididymal tuberculosis mostly occurs in the tail of the epididymis [17], while other studies revealed that epididymal tuberculosis mostly involves the whole epididymis or head [18]. In the present study, tuberculosis lesions were 
mostly found in the caudal epididymis. Previously, it has been widely considered that epididymal tuberculosis is predominantly bilateral, while at present, it is mostly unilateral. In the present study, eight cases (66.7\%) were unilateral, while four cases (33.3\%) were bilateral. Epididymal tuberculosis can be accompanied by pathological changes, such as granulation tissue formation, caseous necrosis and fibrosis, resulting in complex and diverse acoustic images [19]. In the present group of cases, three cases with sinus formation all had testicular involvement.

Ultrasound is the first choice for epididymis imaging examination, and can display the size, shape, structure, boundary and other characteristics of the epididymis. Color Doppler ultrasound can show the distribution of blood flow in the epididymis. Ultrasonic guidance enables a safe, effective and accurate sampling, while avoiding blood vessels and necrotic tissues, making it an ideal method for the biopsy of epididymal tuberculosis. In the present experiment, the pathological features were obtained by ultrasound guided puncture, and the samples were taken for laboratory examination. The diagnostic sensitivity, specificity and AUC value of pathology was $58.33 \%, 100.00 \%$ and 0.79 , respectively, which had good diagnostic value. In terms of safety, ultrasound guided biopsy is performed under direct vision, allowing for the real-time monitoring of the location of the needle tip, the observation of the blood supply of the lesion and necrosis, and the avoidance of the blood vessels to ensure the safety of the puncture. In the present study, the procedure was simple, repeatable, and had no complications.

Laboratory tests are necessary to confirm diagnosis of tuberculosis. GeneXpert MTB/RIF is an automated polymerase chain reaction (PCR) test that accurately detects Mycobacterium tuberculosis and its resistance to Rifampin [20]. This can be used as an alternative test for the routine (including routine microscopy, culture, or histopathology) detection of specific non-respiratory specimens (lymph nodes and other tissues) in patients suspected of extrapulmonary tuberculosis. In comparison to traditional acid-fast staining and tuberculosis culture technology, GeneXpert MTB/RIF is more valuable in the diagnosis of epididymal tuberculosis, which is beneficial for the early detection and timely guidance of the clinical anti-tuberculosis drug treatment. In the present study, the proportional methods for detecting the rifampin resistance on bacterial colonies obtained from two tuberculosis culture positive cases were found to be consistent with the results of the GeneXpert MTB/RIF method. The diagnostic value of GeneXpert MTB/RIF was also demonstrated through its superior AUC value, when compared to other methods, although the test consistency was insufficient due to the rarity of epididymal tuberculosis, and the small number of cases included in the present study.

\section{Conclusion}

Ultrasound guided percutaneous biopsy in combination with GeneXpert MTB/RIF assay has great value in the diagnosis of epididymal tuberculosis and detection of rifampicin resistance, due to its high safety, sensitivity, specificity and superior AUC value.

\section{Declarations}

\section{Ethics approval and consent to participate}

All authors attest that they meet the Helsinki declaration. All methods were carried out in accordance with relevant guidelines and regulations. All experimental protocols were approved by the ethics committee of Shandong Public Health Clinical Center.

\section{Consent for publication}

All authors clarify that the patient's explicit informed consent has been obtained at the time of puncture, and release any identification information contained in each case studied in the manuscript to obtain their informed consent.

\section{Availability of data and materials}

The datasets used and/or analysed during the current study are available from the corresponding author on reasonable request.

\section{Competing interest}


The authors declare that they have no competing interest.

\section{Funding}

Organization: Shandong health and health Committee; Entry name:Shandong medical and health science and technology development plan project; Project number:2019WS535; Project Leader: Qinghu Yan

\section{Authors' contributions}

All authors attest that they meet the current International Committee of Medical Journal Editors (ICMJE) criteria for Authorship.

\section{Acknowledgements}

The authors gratefully acknowledge the financial supports by the Foundation of Shandong health and health Committee under project number 2019WS535. We thank Medjaden Inc. for scientific editing of this manuscript.

\section{Statement for informed consent}

Informed consent was waived by the Ethics Committee of (Shandong Public Health Clinical Center /IRB 2021XKYYEC-22) for this study due to retrospective nature

\section{References}

1. Lamichaney R, Das D, Sherpa M. Kocsh' Disease Presenting as an Isolated Testicular Mass- An Unusual Occurance. J Clin Diagn Res. 2014 Sep; 8(9): FD13-15.

2. Figueiredo AA, Lucon AM. Urogenital tuberculosis: update and review of 8961 cases from the world literature. Rev Urol. 2008; 10(3): 207-217.

3. Dr Keyur NS, Dr Kaushal DS, Dr Jainam KS. Isolated Tuberculous EPididymo-Orchitis: A Rare and Instructive Case RePort[J]. Seajcrr,2012; I(3): 46-50.

4. Liu Xuefeng, Wen Ning, Wu Bin. Clinical diagnosis and treatment of epididymal tuberculosis[J]. Journal of Practical Diagnosis and Therapy, 2006, 20 (11): 822.

5. Desai U, Joshi JM. Extrapulmonary drug-resistant tuberculosis at a drug-resistant tuberculosis center, Mumbai: our experience-hope in the midst of despair! Lung India Off Organ Indian. Chest Soc. 2019; 36(1): 3.

6. Golden MP, Vikram HR. Extrapulmonary tuberculosis: an overview. Am Fam Physician. 2005; 72(9): 1761-1768.

7. Dell'Atti L. Unusual isolated tubercolous epididymitis-Case report[J]. G Chir, 2014; 35(5囚6): 134囚136.

8. Kuhn AL, Scortegagna E, Nowitzki KM, et al. Ultrasonography of the scrotum in adults[J]. Ultrasonography, 2016, 35(3): 180 ه197.

9. Rice J P, Seifert M, Moser K S, et al. Performance of the Xpert MTB/RIF assay for the diagnosis of pulmonary tuberculosis and rifampin resistance in a low-incidence, high-resource setting[J]. PLos One, 2017; 12(10): e0186139.

10. WHO: Tuberculosis (TB). https://www.who.int/tb/areas-of-work/laboratory/en/

11. ChineseMedicalAssociation. Clinical diagnosis standardof TB for clinicaltechnologyoperation(TBvol-umes). People's Medical Publishing House; 2005.

12. China Anti Tuberculosis Association Foundation Specialized Committee. Diagnostic procedures for tuberculosis diagnostic laboratory [M]. Beijing: China Education and Culture Press, 2006, 34-35.

13. Telenti A, Imboden P, Marchesi F, et al. Detection of rifampicin-resistance mutations in Mycobacterium tuberculosis[J]. Lancet, 1993, 341(8846): 647-650.

14. Yang DM, Chang MS, Oh YH, et al. Chroinc tuberculous epididymit is color Doppler US findings with histopathologic correlation[J]. Abdominal Imaging, 2002; 25(5): 559ه562. 
15. Seo JW, Park CJ, Kim TK, et al. Testicular tuberculosis in multidrug-resistant pulmonary tuberculosis. J Infect Chemother. 2013; 19(4): 767-769. doi:10.1007/s10156-012-0508-2.

16. Das A, Batabyal S, Bhattacharjee S, Sengupta A. A rare case of isolated testicular tuberculosis and review of literature. J Fam Med Prim Care. 2016; 5(2): 468. doi:10.4103/2249-4863.192334

17. Kim SH, Pollack HM, Cho KS, et al. Tuberculous epididymitis and epididymoorchitis: sonographic findings. J Urol, 1993, 150 (1) : 81-84.

18. Malai M, Wilfred CG, Bannakit L, et al. Tuberculous epididymitis and epididymo-orchitis: sonographic appearances. AJR Am J Roentgenol, 2001; 176( 6): 1459-1466.

19. YU Liang, Xue Ensheng, Lin Liwu, et al. Value of color Doppler ultrasonography in treatment of epididymal tuberculosis [J/CD]. Chinese Journal of Medical Ultrasound (Electronic Edition), 2008, 5 (2): 303-308.

20. Steingart K R, Sohn H, Schiller I, et al. Xpert MTB/RIF assay for pulmonary tuberculosis and rifampicin resistance in adults. [J]. Cochrane Database of Systematic Reviews, 2014(1):CD009593.

\section{Figures}

(A)

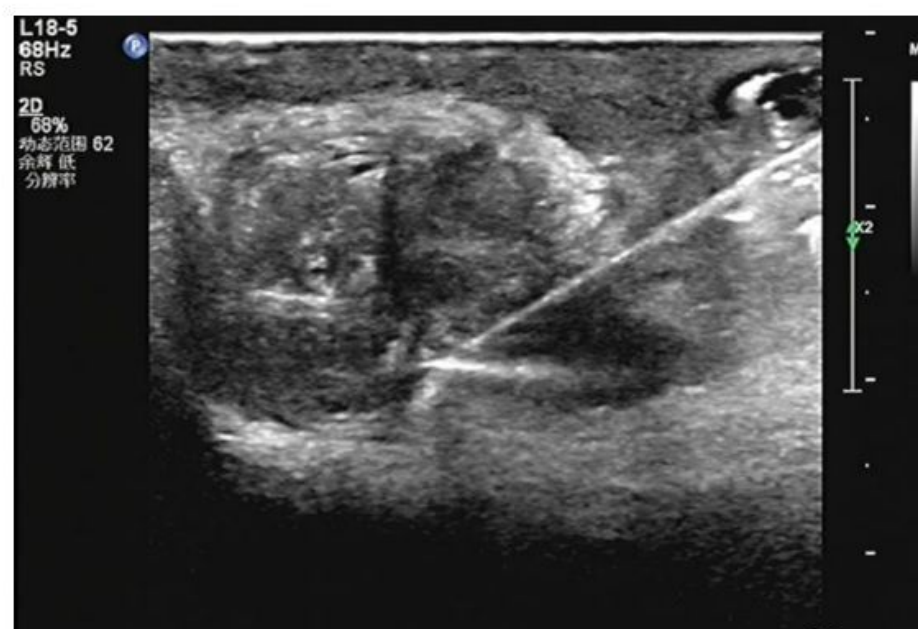

(B)

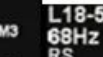

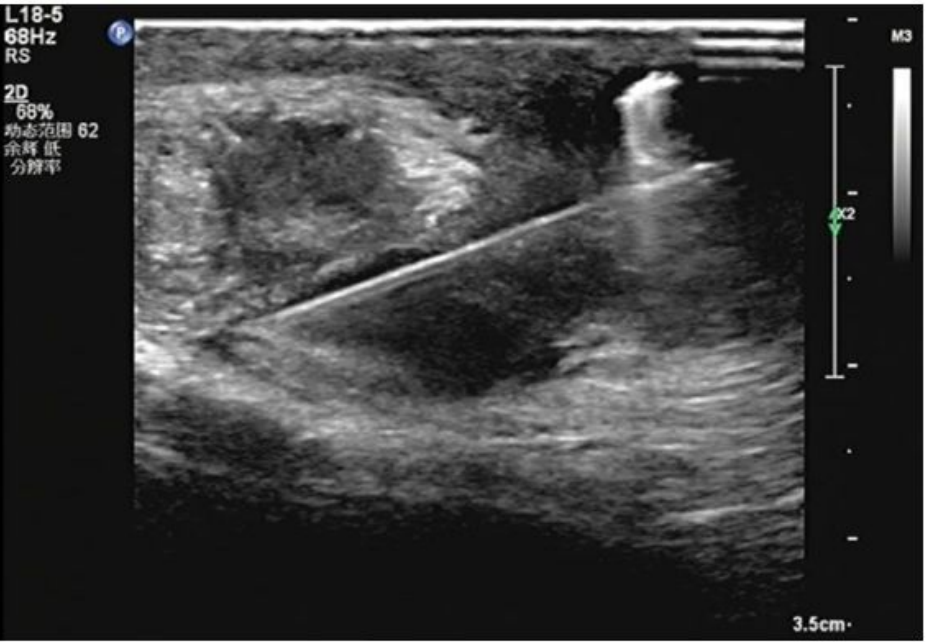

\section{Figure 1}

$(A)$ and $(B)$ : The representative ultrasound imaging clearly reveals the hypoechoic nodules in the epididymis tail, local rupture and scrotum involvement. The ultrasound also clearly directed the puncture needle when performing the biopsy at the focus. 


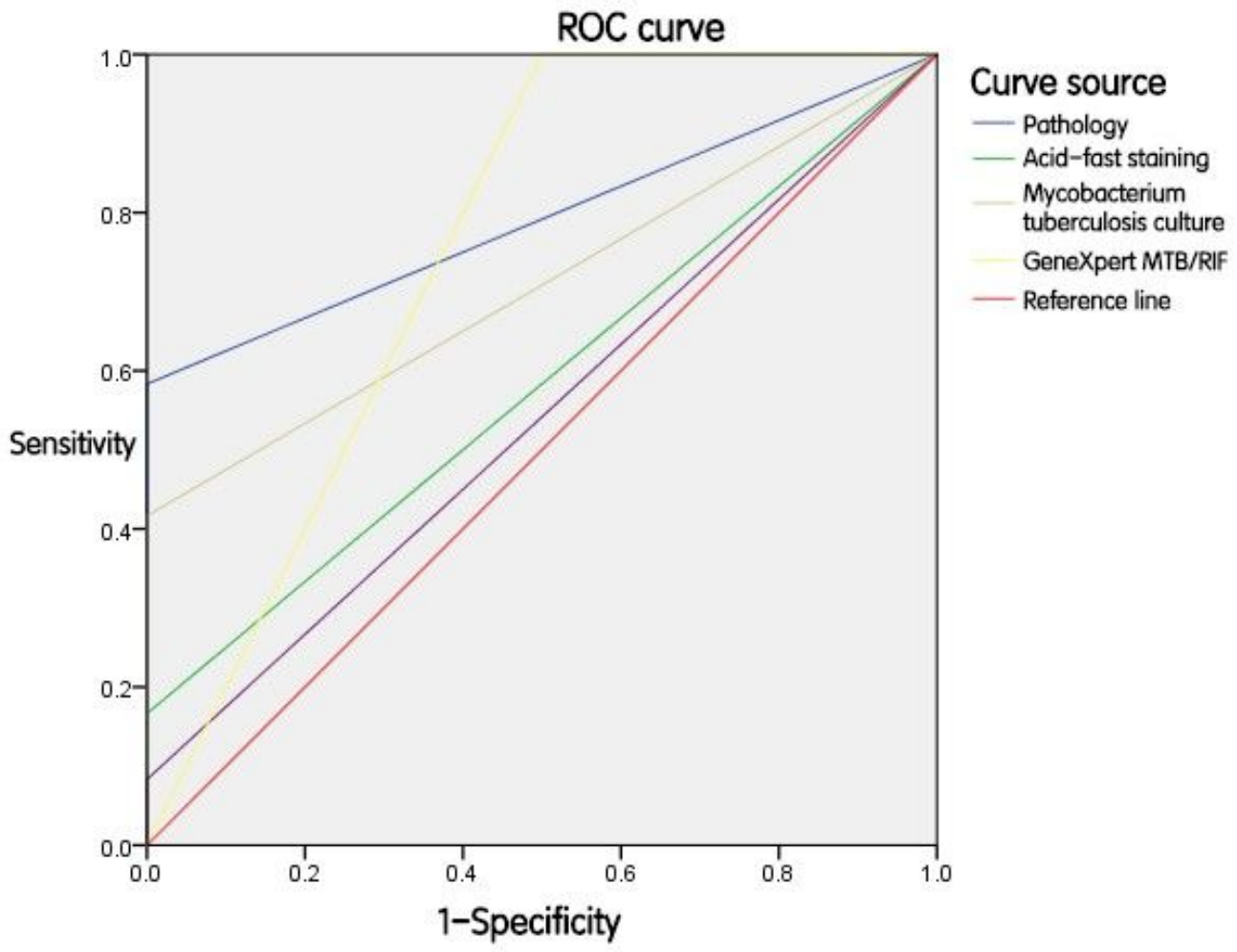

Figure 2

ROC curves of Pathology, Acid-fast staining, Mycobacterium tuberculosis culture, GeneXpert MTB/RIF and Reference line. 